\title{
Es preciso que los pediatras generales mejoren en el diagnóstico de los soplos cardíacos
}

Accuracy of clinical assessment of heart murmurs by office based (general practice) paediatricians. Haney I, Ipp M, Feldman W, McCrindle BW. Arch Dis Child 1999: 81: 409-412

\section{Objetivo}

Determinar la eficacia diagnóstica del examen físico realizado por pediaträs generales en la evaluación de soplos cardíacos.

\section{Diseño}

Estudio de corte transversal

\author{
Lugar \\ Hospital for Sick Children, Toronto, Canadá
}

\section{Pacientes}

Se evaluaron 37 niños asintomáticos con un soplo cardíaco. Los mismos fueron seleccionados del consultorio de dos cardiólogos infantiles y dos pediatras generales en el Hospital for Sick Children.

\section{Descripción de los tests}

Se evaluó el examen físico cardiológico de manos de 30 pediatras generales, la mayoría graduados en Canadá, con residencia completa y que participaron voluntariamente en el estudio. La edad media fue de 53 años. Estos pediatras fueron asignados al azar a tres grupos en los que se evaluaban entre cinco y nueve pacientes. Luego del exámen registraban sus hallazgos e impresión diagnóstica (presencia o no de lesión cardíaca, tipo de patología, y grado de seguridad en su diagnóstico presuntivo).

Fuente de financiamiento: No referida

El test de referencia fue la evaluación por un cardiólogo infantily un ecocardiograma.

\section{Resultados principales}

De los 37 pacientes, 21 tenían soplos inocentes (57\%). Dieciseis niños (47\%) tenían soplos patológicos. Las cardiopatías incluyeron comunicación interventricular (5 pacientes), estenosis valvular pulmonar (4), estenosis valvular aórtica (3), estenosis subaórtica (2), válvula aórtica bicúspide (1), e insuficiencia mitral (1).

De un total de 191 evaluaciones, 142 fueron clasificadas correctamente (74\%). La sensibilidad media fue de $82 \%$, con una especificidad media de $72 \%$. La concordancia inter-examinadores fue muy baja, con un valor de kappa de 0.05 . Los pediatras refirieron que hubiesen derivado para una interconsulta con un cardiólogo infantil o realizar un ecocardiograma a 105 de las 191 evaluaciones (54\%), incluyendo a 39\% de los pacientes con soplos inocentes y $77 \%$ de los pacientes con soplos patológicos. Asumiendo que esa conducta lleva al diagnóstico correcto en los pacientes derivados, esta estrategia aumenta la sensibilidad media a $87 \%$ y la especificidad a $98 \%$.

\section{Conclusiones}

La precisión diagnóstica de la evaluación de niños asintomáticos con soplos cardíacos a cargo de pediatras generales es subóptima. Se necesitan estrategias educacionales para mejorar la precisión y reducir el número de interconsultas innecesarias.

\section{COMENTARIO}

Aproximadamente un $80 \%$ de niños sanos presentan soplos cardíacos en algún momento de su infancia/ adolescencia. Por otro lado, sólo el $0.8 \%$ de los niños nacen con alguna cardiopatía congénita, de las cuales por lo menos dos tercios son sintomáticas. Es decir, cuando un niño sano presenta un soplo, la probabilidad de que tenga una cardiopatía es alrededor del $0.4 \%$. El presente estudio evalúa la precisión diagnóstica del examen físico en manos de pediatras generales. Si bien existe una comparación independiente y ciega con el estándar de referencia (ecocardiografía), tanto el espectro de pacientes como la elección de los examinadores merecen algún comentario. En cuanto a los pacientes elegidos, existe un elevado porcentaje de niños con soplos patológicos con relación a la población general ( $43 \%$ vs $0.4 \%$ ). Si bien la sensibilidad y la especificidad de un test no varían con la prevalencia, sí lo hacen los valores predictivos positivo y negativo. En la población general el valor predictivo negativo es mucho mayor que en la muestra del estudio. En cuanto a los pediatras que intervinieron, se trata de un determinado grupo con formación similar, que participaron de manera voluntaria en el estudio, lo cual limita la generalización de los resultados.

En cuanto a los resultados, se informa el promedio de la sensibilidad y especificidad, existiendo una gran dispersión. No se reporta la existencia de "outliers", aunque sí el coeficiente de correlación kappa, de 0.05, es decir, a pesar de la aparente homogeneidad del grupo, la concordancia entre los examinadores es similar a la que se hubiera encontrado por azar. Tomando los valores medios reportados de sensibilidad y especificidad se pueden calcular los coeficientes de probabilidad positivo y negativo (likelihood ratios), obteniendose valores de 2.9 y 0.25 respectivamente. Es decir, el resultado de la evaluación médica agrega poco a la probabilidad pre-test de

\section{Dr. Gonzalo Mariani}

Departamento de Pediatría. Hospital Italiano de Buenos Aires

\section{Referencias}

1. Swenson JM, Fister DR, Miller SA, Boyle GJ, Ettedgui JA, Beerman LB. Are chest radiographs and electrocardiograms still valuable in evaluating new pediatric patients with heart murmurs or chest pain? Pediatrics 1997; 99: 1-3

2. Birkebaek NH. Hansen LK, Elle B, Andersen PE, Friis M, Egeblad M, Damgaard-Pedersen K, Nielsen NT, 0xhoj H. Chest roentgenogram in the evaluation of heart defects in asymptomatic infants and children with a cardiac murmur: reproducibility and accuracy. Pediatrics 1999; 103 (2): e15-18

3. Danford DA, Nasir A, Gumbiner C. Cost assessment of the evaluation of heart murmurs in children. Pediatrics 1993; 91: 365-8

4. Rosenthal A. How to distinguish between innocent and pathologic murmurs in childhood. Pediatrics Clinics of North America 1984;31 (6): 1229-1240

5. McCrindle BW, Shaffer KM, Kan JS, Zahka KG, Rowe SA, Kidd L. Cardinal clinical signs in the differentiation of heart murmurs in children. Arch Pediatr Adolesc Med 1996; 150: 169-70

tener una cardiopatía asintomática. Si partimos de una probabilidad de so列 probabilidad post-testa $1 \%$; un resultado negativo llevaría la probabilidad ost-test a $0.1 \%$. El uso de estudios complementarios como la radiografía de torax y el electrocardiograma es controvertido, habiendo consenso de que contribuye poco al diagnóstico en estos $\operatorname{casos}^{1-2}$.

En estos tiempos de economización de recursos me parece oportuno comentar que la derivación al cardiólogo no debería necesariamente implicar la realización de un ecocardiograma, si bien estos dos términos (interconsulta cardiológica y ecocardiograma) ultimamente se han convertido en sinónimos. Un estudio mostró que la realización directa de ecocardiografía era una forma cara de evaluar a los niños con soplos en comparación con una consulta con un cardiólogo infantil ${ }^{3}$.

Los resultados del presente estudio pueden llevar a conductas en mi opinión erróneas. Es decir, si se concluye que existe un error demasiado alto sobre todo en niños con soplos patológicos que son subvalorados,esto podría llevar a aumentar excesivamente las derivaciones (interconsultas) al cardiólogo infantil. Las características de los soplos inocentes han sido bien definidas ${ }^{4-5}$ y deben ser tenidas en cuenta por los pediatras o médicos generalistas. A la luz de los resultados de este estudio parecería ser necesario implementar estrategias para mejorar las habilidades diagnósticas de los médicos generalistas.

Creo que la formación y experiencia del médico en evaluación cardiovascular, el nivel de cooperación del niño durante el examen físico, la ansiedad de los padres, y la posibilidad de un seguimiento ambulatorio adecuado determinarán si se justifica la realización de estudios adicionales y/o derivación al cardiólogo. 